Nouvelles perspectives en sciences sociales

\title{
Avant-propos
}

\section{Mélanie Girard}

Volume 15, numéro 1, novembre 2019

Sur le thème du tatouage

URI : https://id.erudit.org/iderudit/1068177ar

DOI : https://doi.org/10.7202/1068177ar

Aller au sommaire du numéro

\section{Éditeur(s)}

Prise de parole

\section{ISSN}

1712-8307 (imprimé)

1918-7475 (numérique)

Découvrir la revue

Citer ce document

Girard, M. (2019). Avant-propos. Nouvelles perspectives en sciences sociales, 15(1), 15-18. https://doi.org/10.7202/1068177ar d'utilisation que vous pouvez consulter en ligne.

https://apropos.erudit.org/fr/usagers/politique-dutilisation/ 


\section{Avant-propos}

\section{MÉLANIE GIRARD}

Université de Hearst

T e numéro que voici aborde la thématique des modifications corporelles. Il le fait surtout du point de vue du tatouage, bien que l'appel à communication sollicitait des contributions sur toutes les formes de modifications corporelles, qu'il s'agisse de tatouage, d'implants subdermiques, d'étirement, de scarification, de chirurgie esthétique, voire de mutilations sexuelles, par exemple. Il nous semblait que le moment était venu de se pencher sur la question des bod mods compte tenu du déploiement qu'elles connaissent depuis un peu plus de trois décennies et des nombreuses questions qu'elles soulèvent. Objet intrinsèquement interdisciplinaire, les modifications corporelles confrontent en effet à des considérations tant éthiques ou morales que scientifiques ou juridico-politiques, par exemple. À la confluence des sciences humaines et naturelles, mobilisant les acquis et sollicitant les outils des sciences de la communication, des sciences du vivant et de la santé, de l'art, de la psychologie, de la géographie, de l'histoire et de l'anthropologie, du marketing ou de l'économie, de la littérature et de la sémiologie, notamment, elles obligent à une prise en compte, dans le regard qu'on leur conférera, de cette inhérente complexité qui caractérise le rapport 
entre l'humain et la peau qu'il habite et, conséquemment, dont est porteur le geste de modifier son corps, qu'il soit ou non volontaire.

Nous avons voulu conférer aux modifications corporelles toute la place qu'elles occupent, qu'elles ont occupé dans l'histoire de la civilisation occidentale. Ainsi, c'est sous toutes ses facettes que nous proposions d'étudier le corps : comme objet d'art ou de revendication politique, comme siège et comme exutoire des passions et du vice, comme théâtre de la fertilité et de la vie, comme frontière entre soi et le monde... rappelant ainsi le lien intime entre corps et identité et ouvrant la voie aux réflexions sur le corps transformé, modelé, voire augmenté, bref sur le corps prêt-à-perforer-tatouer-scarifier-marquer-suspendre-implantertransplanter-occulter, mais aussi, sur le corps marqué ou souillé, c'est-à-dire sur le corps qui porte les traces d'un passé dont on souhaite se distancier.

Dans le premier texte, "Corpus religiosum: La question des marques corporelles dans le christianisme latin ", Jean-Pierre Albert s'intéresse à la stigmatisation religieuse dans le contexte du christianisme latin ; il montre comment les ancrages corporels d'un habitus chrétien permettent d'appréhender le phénomène en tenant compte à la fois des déterminants anthropologiques généraux du religieux et des spécificités de la théologie chrétienne. Il en vient ainsi à distinguer entre trois rapports au corps dans le contexte de la chrétienté : la honte du corps sexué qui émane des pratiques chrétiennes liées au corps, les preuves par le corps de la sainteté qui se dégagent des pratiques dévotionnelles, la souffrance dont font état les empreintes surnaturelles, lesquelles constituent un trait universel de l'engagement religieux du corps, soit le lien entre marque corporelle et douleur physique.

Partant du corps en tant qu'espace, Simone Wiener présente "Le tatouage comme archive, de la trace de l'écriture à l'écriture de la trace ", suggérant ainsi que le tatouage, symptomatique de notre époque, aurait une fonction d'ancrage : en tant que trace visible, il permettrait d'inscrire le corps dans un espace à la fois physique et symbolique, personnel et partagé. Passant sous 
révision les aspects traditionnels du tatouage et distinguant entre tatouages tribaux et totémiques, l'autrice explore différentes formes d'inscription corporelle, du stigmate lié à un projet génocidaire au symbole imagé qui permet la ré-identification au corps. C'est à travers une perspective psychanalytique qui pose le corps comme frontière entre soi et l'Autre et, plus précisément, en positionnant le tatouage comme participant de la pulsion scopique qu'elle arrive à faire valoir, au-delà de l'inscription personnelle, la portée collective du tatouage, dont le sens ne saurait s'extraire du regard de l'Autre.

Puisant elle aussi dans la psychanalyse, dans son texte " De la scarification au tatouage : une écriture intime », Catherine Rioult présente le tatouage comme outil de sublimation de pulsions destructrices. Rappelant la fonction anthropologique du tatouage dans son rapport avec l'identité, elle montre, à travers le parcours psychothérapeutique d'adolescentes, comment la peau peut servir de support à une écriture intime par laquelle elles conjurent un mal de vivre, passant ainsi de scarifications infligées dans le secret et la douleur à un tatouage choisi et assumé aux yeux de tous qui, bien qu'intime, se veut aussi public.

Dans « Représentations du tatouage : le paradoxe de la différenciation et de l'assimilation » Mélanie Girard, Simon Laflamme et Claude Vautier explorent explicitement la tension entre individuel et collectif. S'intéressant à la montée en popularité du tatouage au cours des quelques dernières décennies, les auteurs suggèrent qu'il aurait pour fondement une épistémè qui prend racine dans quatre phénomènes distincts, mais interreliés : le rapport à l'espace, la montée en puissance des super héros, la révolution sexuelle, l'apparition d'internet. À travers les données d'une enquête franco-canadienne et en utilisant des logiciels d'analyse de données textuelles, ils montrent que le fait de se faire tatouer ne peut être qu'un geste à la fois individuel et collectif qui s'arrime à des considérations sur l'art et l'esthétique, sur la santé, sur les normes sociales et sur les limites physiques et psychologiques, que l'on soit Français ou Canadien, que l'on soit non tatoué, peu tatoué ou très tatoué. 
Dans son texte "Se détatouer : une furtivité ou une vivacité corporelle ? ", Bernard Andrieu s'intéresse au détatouage et il suggère qu'il pourrait être compris comme un effacement : effacer son passé pourrait être le moyen de recommencer à zéro, de retrouver une peau d'origine et de donner un sens nouveau à sa peau. Cependant, examinant les techniques d'effacement, il met en valeur leur caractère invasif et, ainsi, le désengagement qui incombe à l'individu qui choisirait de se soumettre aux séquelles qu'elles sous-tendent ; en ce sens, il suggère qu'il n'y a pas un arrangement, mais bien un agenrement à opérer avec sa peau pour redonner un style et un genre à un corps déconsidéré par ce qui serait maintenant un défaut à éliminer. 